\title{
Single-phase-to-ground fault line selection in distribution networks based on similarity of wavelet energy spectrum
}

\author{
Mingjun $\mathrm{Qi}^{1}$, Qiang Zhong ${ }^{2, \mathrm{a}}$, Liang Zhu ${ }^{1}$, Fangliang Gong $^{1}$, Hua Leng ${ }^{1}$ \\ ${ }^{1}$ State Grid Hunan Electric Power Company, Changsha 410007, China \\ ${ }^{2}$ College of Electrical and Information Engineering, Hunan University, Changsha, 410082, China \\ aemail: zhongqiang1225@gmail.com
}

Keywords: fault line selection; wavelet packet analysis; similarity of energy spectrum

\begin{abstract}
In order to solve the problems of ground fault line selection in distribution network, the article presents a fault line selection method based on the similarity of the wavelet packet energy spectrum. After extracting a certain data of transient fault zero-sequence current by the wavelet packet decomposition, we can get the energy spectrum distribution and energy spectrum matrix of all lines. Comparing the similarity of the energy spectrum distribution of all lines, a minimum similar principle as the criterion of the fault line is selected. Simulation results verified that this method can accurately and fast select the fault line with good adaptability.
\end{abstract}

\section{Introduction}

In china, distribution network system uses non-effective grounding manner in most cases, while the single-phase ground fault line in small current grounding has been not been completely resolved [1]. Domestic and foreign scholars have done a lot of research work in the fault line detection. Many scholars have proposed approach based on the fault line current steady-state volume or the transient-state current volume. Literature[2][3] proposed the method using the transient-state current volume, but unstable and difficult to extract transient signals, so the method using transient current can't be well applied. Literature[4-10] use wavelet analysis of signal processing, using band energy as the fault feature to provide the main criteria for the selection of the line. While the difference of energy concentration bands between feeder lines is large, the method may cause false positives.

This paper presents a fault line selection method based on the difference between the energy spectrums of each feeder lines after the wavelet packet decomposition. This method makes full use of information of each band. This method is not affected by arc suppression coil, high-resistance grounded and arc-grounding.

\section{The transient analysis of zero sequence current in single-phase-to-ground fault}

When analyzing the dynamic circuit theory, we regard the single-phase-to ground fault as the switch to open and close with the added virtual power[11]. Therefore, the analysis of single-phase ground fault transient process can use the equivalent circuit shown in Fig 1.

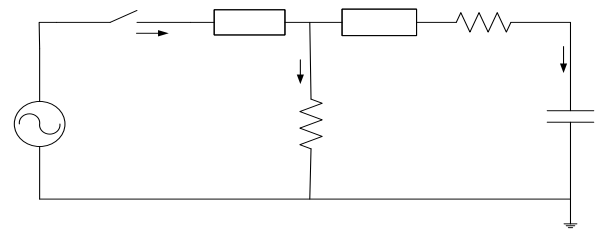

Fig.1. Equivalent circuit of single-phase-to-ground fault

When single-phase-to-ground fault occurs, according to the theory of the second-order circuit zero-state response, you canget the capacitive loop equation. 


$$
\begin{aligned}
& i_{0 C}\left(3 R_{t r}+R\right)+L \frac{d i_{0 C}}{d t}+\frac{1}{C_{0}} \int_{0}^{t} i_{0 C} d t+u_{C}\left(0_{-}\right)=U_{m} \sin (\omega t+\psi)(1) \\
& i_{0 C}=I_{C m}\left(\frac{\omega_{f}}{\omega} \sin \psi \sin \omega_{f} t-\cos \psi \cos \omega_{f} t\right) e^{-\delta t}+I_{C m} \cos (\omega t+\psi)(2)
\end{aligned}
$$

According to the Laplace transform derived transient expression for the instantaneous inductor current is

$$
i_{0 L}=I_{L m} \cos \psi e^{-\frac{t}{\tau_{L}}}-I_{L m} \cos (\omega t+\psi)(3)
$$

To give a fault current flowing in the ground is

$$
i_{k}=i_{k . s}+i_{k . t}=\left[I_{C m} \cos (\omega t+\psi)-I_{L m} \cos (\omega t+\psi)\right]+\left[I_{C m}\left(\frac{\omega_{f}}{\omega} \sin \psi \sin \omega_{f} t-\cos \psi \cos \omega_{f} t\right) e^{-\delta t}+I_{L m} \cos \psi e^{-\frac{t}{\tau_{L}}}\right)
$$

Through the above analysis, transient ground current is mainly composed of the steady-state component and a transient component[10].By the Eq.(4),The main factors that influence the zero sequence current are the initial phase angle, ground over impedance and line parameters. These fault conditions have the impact of transient zero sequence current being coupled.

\section{Wavelet packet transform principle}

Wavelet packet analysis has a very good signal response feature in the field of time-frequencydomain, very suitable for the ex-traction of zero-sequence current transient characteristics[12].

We can define that the subspace is the closure spaces of function, and is the closure spaces of function. These must meet the following two-scale equation[13].

$$
\begin{aligned}
& \mathrm{u}_{2 \mathrm{n}}(t)=\sqrt{2} \sum_{k} h(k) \mathrm{u}_{\mathrm{n}}(2 t-k)(5) \\
& \mathrm{u}_{2 \mathrm{n}+1}(t)=\sqrt{2} \sum_{k} \mathrm{~g}(k) \mathrm{u}_{\mathrm{n}}(2 t-k)(6)
\end{aligned}
$$

Among the equations, $\mathrm{h}(\mathrm{k})$ and $\mathrm{g}(\mathrm{k})$ are the coefficients of wavelet packet filter, having orthogonal. When selecting the wavelet function, you must select orthogonal wavelet. Dubeties $(\mathrm{dbN})$ wavelet always be applied to engineering.

Base on wavelet packet frequency characteristics, the input signal is decomposed into sub-bands under $\mathrm{j}$ decomposition standard. After divided, each frequency band corresponds to the following expression[14].

$$
E_{j}^{i}=\sum^{n}\left[c_{i}^{j}(n)\right]^{2}(7)
$$

$c_{i}^{j}(n)$ is the coefficient of wavelet packet divided into $(\mathrm{j}, \mathrm{i})$ sub-band. Every sub-band has $\mathrm{n}$ coefficients and $\mathrm{j}$ is the measure of wavelet decomposition, iis thei-thnode of wavelet decomposition. The fig2 shows that the polarity of fault line and non-fault line zero-sequence current is different as single phase ground is fault, and there is a big difference in their energy distribution as showed in fig3, which provide effective fault feature for the fault line selection.

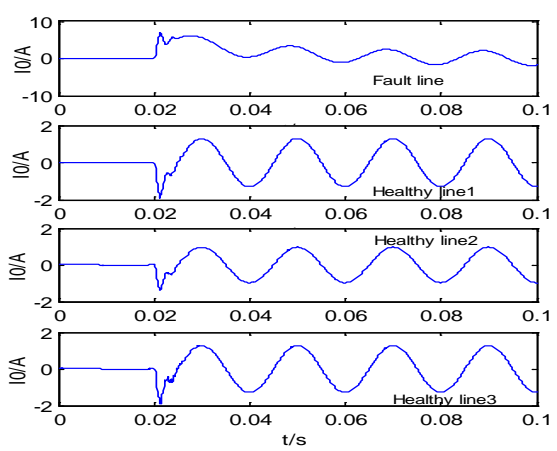

Fig.2. Transient zero-sequence current
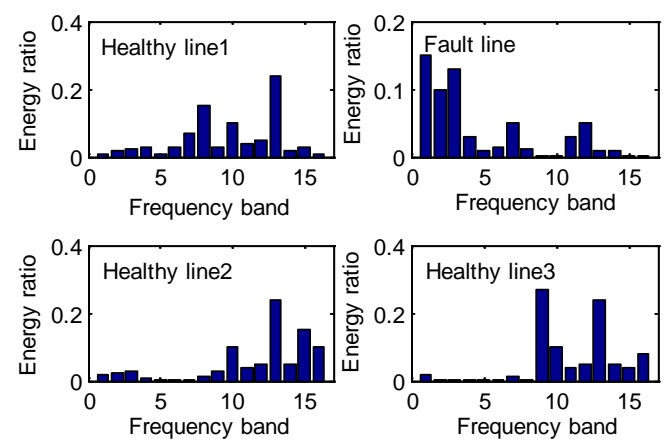

Fig. 3. Energy ratio of fault line and healthy lines 
Signals of fault line and healthy lines

Through spectrum analysis to fault current, we can see that the wavelet decomposition making use of distinguish analysis can decompose the signal in the time -frequency domain and effectively extract fault characteristics. In the case of large scale decomposition, a lot of band information is abandoned. This approach will lead to the loss of much information, which results in decline of line selection margin. According to the thought of energy spectrum combined with image processing similarity, a new fault line selection method based on energy spectrum similarity is born.

According to the equation (7), we decompose the each line under the $\mathrm{j}$ criterion and then get every wavelet packet reconstruct coefficient. Then we decompose reconstruct coefficient of each band, and divide $\mathrm{n}$ coefficient of each sub-band into $\mathrm{M}$ equal portions, which generally is matrix. The more sophissticated quotation under (j, i) band is

$$
E_{m}^{n}=\sum^{M}\left[c_{m}^{n}(k)\right] \Delta T \quad \text { (8) }
$$

$c_{m}^{n}(k)^{k=1}$ is the k-th reconstructed coefficient of wavelet packet divided into jsub-band. Each sub-band has the same coefficient. $\Delta T$ is the time of every aliquot. Through the quotation (8), we can get the wavelet energy spectrum matrix $\mathrm{E}$.

$$
E=\left[\begin{array}{cccc}
E_{1}^{1} & E_{1}^{2} & \ldots & E_{1}^{m} \\
\ldots & \ldots & \ldots & \ldots \\
E_{n-1}^{1} & E_{n-1}^{2} & \ldots & E_{n-1}^{m} \\
E_{n}^{1} & E_{n}^{2} & \ldots & E_{n}^{n}
\end{array}\right](9)
$$

Based on the thought of using of digital image processing to graphics similarity, $E_{a} E_{b}$ are set fault line energy matrix and non-fault line energy matrix. The similarity can shows that

$$
M_{a b}=\sum_{m=1}^{M} \sum_{n=1}^{N}\left[E_{a}(m, n)-E_{b}(m, n)\right]^{2}
$$

We can get the similarity define after normalizing the quotation[15].

$$
S_{a b}=\frac{\sum_{m=1}^{M} \sum_{n=1}^{N} E_{a}(m, n) E_{b}(m, n)}{\sqrt{\sum_{m=1}^{M} \sum_{n=1}^{N}\left[E_{a}(m, n)\right]^{2} \sum_{m=1}^{M} \sum_{n=1}^{N} E_{b}(m, n)}}
$$

$\mathrm{M}$ of quotation (10) and $\mathrm{N}$ of quotation (11) respectively represent the number of maxim columns and the number of maxim rows. In the energy spectrum matrix, they respectively represent the number of wavelet packet decomposition bands and periods. In order to improve the margin line selection, the comprehensive similarity coefficient of each line $I(i=1,2,3 \ldots n)$ is defined that

$$
\rho_{i}=\frac{1}{n-1}\left(\sum_{j=1, j \neq i}^{n} S_{i j}\right)(12
$$

Because the fault feeder and non-fault feeder have energy distribution differences under wavelet decomposition, the line selection method of energy spectrum similarity is proposed. When the fault voltage exceeds the bus bar rated voltage of 0.35 , it will start to record the zero-sequence current of each outlet. The sampling frequency is $10 \mathrm{KHz}$. We should use db10 wavelet to decompose and reconstruct to five layers, and divide the wavelet packet coefficient reconstructed into the same part using the time standard. So we can respectively get each of the emerging spectrum matrixes E, and get comprehensive similarity coefficient $\rho$ of each line according to their degree of similarity. The energy values are positive, and the energy spectrum similarity of experimental validation failures and non-fault lines don't exceed 0.2 . The threshold value of $\rho_{\text {set }}$ is 0.3 . Low degree of similarity is the fault line, that is to say, when $\rho_{\mathrm{i}}<\rho_{\text {set }}$, the $\mathrm{i}$-th line appears fault. If all the value of $\rho$ are greater than the threshold value, it is bus fault.

\section{Simulation analysis}

The literature[4] provides the distribution network model. Using Matlab power system simulation module to build a $110 \mathrm{kV} / 10 \mathrm{kV}$ large capacity ground fault model shown in Figure 
4 .The model has six stages, the line length was $3 \mathrm{~km}, 6 \mathrm{~km}, 9 \mathrm{~km}, 12 \mathrm{~km}, 15 \mathrm{~km}, 20 \mathrm{~km}$. The positive sequence impedance is $Z_{1}=(0.17+j 0.38) \Omega / \mathrm{km}$, positive sequence admittance is $b_{1}=j 3.045 \mu \mathrm{S} / \mathrm{km}$, Zero sequence impedance is $Z_{0}=(0.23+j 1.72) \Omega / \mathrm{km}$, Ground zero sequence admittance is $b_{0}=j 1.884 \mu \mathrm{S} / \mathrm{km}, \mathrm{G}$ is infinite power, Main transformer turns ratio is coil grounding through, the system uses $8 \%$ over compensation, $110 \mathrm{kV} / 10 \mathrm{kV}$,loadparameters is $Z_{L}=400+j 200 \Omega$, Neutral point arc suppression.

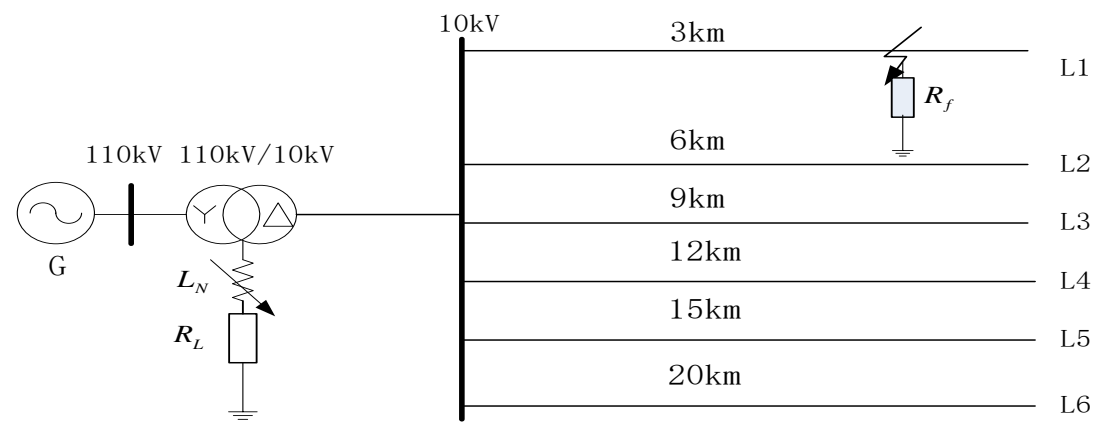

Fig.4. Simulation model

$l$ is the line length, $f_{N}$ is $50 \mathrm{~Hz}, C_{0}$ is feeder zero sequence current, $v$ is detuning degree, $v=\left(I_{C}-I_{L}\right) / I_{C}$,calculated according to the parameters and the length $L_{N}=8.02 \mathrm{H}$, Resistor in series according to $10 \%$ of the Petersen coil inductance values calculated the $R_{L}=252 \Omega$

While the amplitude of ground current is significant, but transient process duration is very short, about 0.5 to 1 frequency cycle. So the choice of one cycle zero sequence current as a fault simulation data, the sampling frequency is $10 \mathrm{kHz}$.Simulation analysis is in different situations, such as different fault location, different fault initial angles and different transition resistance. The following table 1 shows the results of selected lines. $L_{i}$ is the fault lines, $X_{f}$ is the distance from the bus fault, $R_{f}$ is the fault initial angles.

Table 1. Results of fault line detection

\begin{tabular}{|c|c|c|r|c|c|c|}
\hline $\begin{array}{c}\text { Fault } \\
\text { line }\end{array}$ & $X_{f} / \mathrm{km}$ & $R_{f} / \Omega$ & $\left.\theta /{ }^{\circ}\right)$ & $\begin{array}{c}\text { Spectrum similarity coefficients } \\
\left(\rho_{1} \rho_{2} \rho_{3} \rho_{4} \rho_{5} \rho_{6}\right)\end{array}$ & $\mathrm{Y} / \mathrm{N}$ \\
\hline \multirow{4}{*}{$\mathrm{L} 1$} & 1 & 20 & 0 & $0.1274 、 0.8741 、 0.8654 、 0.8456 、 0.8432 、 08932$ & $\mathrm{Y}$ \\
\cline { 2 - 7 } & 2 & 500 & 30 & $0.2224 、 0.7389 、 0.7580 、 0.8045 、 0.7230 、 0.8036$ & $\mathrm{Y}$ \\
\cline { 2 - 7 } & 3 & 1000 & 90 & $0.1164 、 0.7997 、 0.7780 、 0.7345 、 0.7643 、 0.7432$ & $\mathrm{Y}$ \\
\hline \multirow{4}{*}{ L4 } & 2 & 1000 & 0 & $0.8543 、 0.8901 、 0.8538 、 0.2161 、 0.8648 、 0.8149$ & $\mathrm{Y}$ \\
\cline { 2 - 7 } & 8 & 500 & 45 & $0.8650 、 0.8238 、 0.8270 、 0.2035 、 0.8179 、 0.8536$ & $\mathrm{Y}$ \\
\cline { 2 - 7 } & 12 & 20 & 90 & $0.8363 、 0.8222 、 0.8213 、 0.2266 、 0.8205 、 0.8326$ & $\mathrm{Y}$ \\
\hline \multirow{3}{*}{$\begin{array}{c}\text { Bus } \\
\text { bar }\end{array}$} & 0 & 500 & 30 & $0.7987 、 0.7432 、 0.6999 、 07219 、 0.7234 、 0.7321$ & $\mathrm{Y}$ \\
\cline { 2 - 7 } & & 1000 & 90 & $0.7098 、 0.7775 、 0.7732 、 0.7126 、 0.7034 、 0.7444$ & $\mathrm{Y}$ \\
\hline
\end{tabular}

\section{Adaptability Analysis of the methods}

In the distribution network system, grounding fault with high resistance occur with high frequency.Besides,in order to test and verify the capacity of resisting disturbance, this paper obtained large amounts of data by modeling and simulation methods. The simulation results were shown in table2 and table3depending on various situation. 
Tab.2 Results of faulty line selection with noise in line L5

\begin{tabular}{|c|c|c|c|c|}
\hline$\theta /{ }^{\circ}$ & Compensation & $\begin{array}{c}\text { Spectrum similarity coefficients } \\
\left(\rho_{1} \rho_{2} \rho_{3} \rho_{4} \rho_{5} \rho_{6}\right)\end{array}$ & $\mathrm{Y} / / \mathrm{N}$ \\
\hline 0 & Ungrounded & 0.8378、0.8121、0.8222、0.7351、0.1156、0.8034 & $\mathrm{Y}$ \\
\hline 30 & Overcompensation $10 \%$ & $0.8368 、 0.8334 、 0.8215 、 0.8590 、 0.1753 、 0.8426$ & $\mathrm{Y}$ \\
\hline 90 & Undfilled20\% & $0.6324 、 0.6321 、 0.6666 、 0.6987 、 0.2754 、 0.6963$ & $\mathrm{Y}$ \\
\hline
\end{tabular}

Tab3 Results of faulty line selection for grounding fault with high resistance at the end of line L6.

\begin{tabular}{|c|c|c|c|}
\hline$\theta /\left(^{\circ}\right)$ & Compensation & $\begin{array}{c}\text { Spectrum similarity coefficients } \\
\left(\rho_{1} \rho_{2} \rho_{3} \rho_{4} \rho_{5} \rho_{6}\right)\end{array}$ & $\mathrm{Y} / \mathrm{N}$ \\
\hline 0 & Undfilled15\% & $0.9230 、 0.9223 、 0.9201 、 0.9231 、 0.9034 、 0.1101$ & $\mathrm{Y}$ \\
\hline 45 & Overcompensation 8\% & 0.7589、0.7993、0.7423、0.7902、0.7657、0.1276 & $\mathrm{Y}$ \\
\hline 90 & Ungrounded & $0.8882 、 08390 、 0.9032 、 0.8357 、 0.8457 、 0.1754$ & $\mathrm{Y}$ \\
\hline
\end{tabular}

\section{Conclusion}

The ideological of this method is from the spectrum similarity theory. Dividing the signal energy to each band, and then decompose each band into $\mathrm{N}$ equal parts, so as to obtain the energy spectrum matrix and the similarity coefficients of each feeder lines, using similarity smallest as the criterion of fault line selection. Overcome the effects of the small fault angle, the different degree of compensation, and the different lines to the resonant grounding line. As a result of theory and Matlab simulation experiment, it proved this method can be accurately fault Line selection with good adaptability and reliability in many cases, such as transient grounding fault, intermittent arcing grounding fault, grounding fault with noise interference and high resistance grounding fault.

\section{Acknowledgements}

This work is supported by National Natural Science Foundation of China under Grant No.61173108 and 61472128, Hunan Provincial Natural Science Foundation of China No.10JJ2045 as well as $14 \mathrm{JJ} 2150$ and State Grid Corporation Science and technology projectof China No.5216A514001K.

\section{References}

[1]Wang Qingliang; Fault line selection and analysis in Single-phase-to-ground system.China electric power press.Beijing(2013).

[2]Li Bin, Shu Hongchu; A new integration method of fault line detection in resonant earthed system based on instantaneous real power and instantaneous virtual power .Transactions of china electro technical society,27(6):483-190(2012).

[3]Li Sen, Song Guobing,Kang Xiaoning,et al.Time-domain fault line selection based on correlation analysis in neutral indirect ground system[J].Power system protection and control,2008,36(13):15-20.

[4]Wu Lepeng,Huang Chun,Lin Dabin,et al.Faulty line selection based on transient wavelet energy for non-solid-earthed network[J].Electeic power automation equipment,2013,33(5):70-75.

[5]Wang Qingliang,Fu Zouxing.An adaptive method for single-phase-to-ground fault line selection based on energy entropy MEAmeasure[J].Automation of electric power systems,2012,36(5):103-111.

[6]Dai Jianfeng,Zhang Yanxia.Study on adaptively choosing fault line under single-phase to ground fault based on analysis of multi-frequency bands[J].Proceedings of the CSEE,2003,23(5):44-47. 
[7]Li Tianyun,Wang Fei,Zhu Lei,et al.A new method of distribution network single-phase ground fault line selection based on intrinsic mode energy entropy[J].Power system technology,2008,32(2):128-132.

[8]Norman C F.tse,John Y C.Chan.Wing-Hong Lau,et.al.Hybridwavelet and hilbert transform with frequency-shifting decomposition for power quality analysis[J].IEEE Transactions on instru-

mentation and measurement,2012,61(12):3225-3233.

[9]Chen Jikai,Li Haoyu,Yang Shiyan.A method to recognize transient voltage disturbance in power system based on non-extensive wavelet entropy and ant colony optimization[J].Proceedings of the CSEE,2010,30:31-36.

[10]Wang Yangnan,HuoBailin,Wang Hui,et al.A new criterion for earth fault line selection based on wavelet packets in small current neutral grounding system[J].Proceedings of the CSEE,2004,24(6):54-58.

[11]Shu Hongchun; Fault line selection in distribution network.Machinery industry press.Beijin (2008).

[12]Nikolaj Hess-Nielsen,Mladen V W.Wavelets and time-frequency analysis [J].proceedings of the IEEE,1996,84(4):523-540.

[13]He Zhengyou, Qian Qingquan.The electric power system transient signalwavelet analysis method and its application(-)The application of wavelet transform in electric power system transient signal analysis summarizing[J].Proceedings of the EPSA,2002,14(4):1-9.

[14]He Zhengyou, Luo Guomin,Yang Jianwei.Power transients recognition based on wavelet energy matrixes similarity[J].Journal of electric power science and technology,2007,22 (3) :12-17.

[15]GuoMoufa,Liu Shidan,Yang Gengjie.A novel approach to detect fault lines in distribution network using similarity recognition based on time-frequency spectrum[J].Proceedings of the CSEE,2013,33(19):183-190. 